\author{
Aus dem Institut für Radiologie \\ der Medizinischen Fakultät \\ Charité - Universitätsmedizin Berlin
}

\title{
DISSERTATION
}

\section{Cerebral multifrequency MR \\ elastography by remote excitation of intracranial shear waves}

\author{
zur Erlangung des akademischen Grades \\ Doctor rerum medicinalium (Dr. rer. medic.)
}

vorgelegt der Medizinischen Fakultät

Charité - Universitätsmedizin Berlin

von

Diplomphysiker Andreas Fehlner aus Weiden in der Oberpfalz

Datum der Promotion: 09. Dezember 2016 


\section{Inhaltsverzeichnis}

1 Zusammenfassung $\quad 3$

2 Abstract $\quad 5$

$\begin{array}{lll}3 & \text { Eidesstattliche Versicherung } & 7\end{array}$

4 Ausführliche Anteilseignung an der erfolgten Publikation $\quad 8$

5 Auszug aus der Journal Summary List $\quad 9$

6 Druckexemplar der ausgewählten Publikation $\quad 11$

$\begin{array}{llr}7 & \text { Lebenslauf } & 18\end{array}$

8 Publikationsliste $\quad 20$

9 Danksagung $\quad 24$ 


\section{Zusammenfassung}

\section{Hintergrund}

In der Medizin besitzt die Palpation zur Untersuchung von Gewebeveränderungen und somit zur Erkennung von Krankheitsprozessen große Bedeutung. Elastische Gewebeeigenschaften des lebenden Gehirns sind durch Palpation nicht zugänglich. Mit der Elastographie werden mechanische Gewebeeigenschaften unter Verwendung moderner medizinischer Bildgebungsmodalitäten auch im Gehirn nicht-invasiv quantifizierbar. Dazu werden mechanische Wellen im Gehirn angeregt und aus den aufgenommenen Wellenbildern Karten viskoelastischer Gewebekenngrößen berechnet. Das Ziel der Studie bestand darin, eine effektive und gleichzeitig Patienten schonende Mechanik zur Wellenanregung über das Brustbein für die hochaufgelöste zerebrale Multifrequenz-Magnetresonanzelastographie (mMRE) zu entwickeln und als klinisch anwendbare Methode zu validieren.

\section{Methoden}

Bei 12 Probanden wurde die bisherige direkte Wellenanregung über eine Kopfwippe mit der neu entwickelten indirekten Anregungstechnik mittels thorakaler Brustmatte verglichen. Dazu wurde die mMRE mit fünf Anregungsfrequenzen von 25 bis $45 \mathrm{~Hz}$ angewandt. Eine koronare Schichtorientierung wurde gewählt, um verschiedene Regionen entlang des pyramidalen Systems abzubilden. Anhand der gemessenen Scherwellen wurden mittels MultifrequenzInversion hochaufgelöste Karten der viskoelastischen Gewebeparameter $\left|G^{*}\right|$ und $\varphi$ rekonstruiert. Weiterhin wurden die Amplituden der Scherwellen im Gehirn quantifiziert. Komfort und Benutzerfreundlichkeit beider Anregungsmethoden wurden von allen Probanden und den 3 Untersuchern anhand eines Fragebogens beurteilt.

\section{Ergebnisse}

Durch die indirekte mechanische Anregung wurde der Patientenkomfort signifikant verbessert $(p=0,02)$ und der Bedienungsaufwand verringert. Gleichzeitig konnten Schwankungen der Scherwellenamplituden zwischen den Probanden, wie sie mit dem herkömmlichen Anregungskonzept auftreten, verringert werden. Die gemessenen viskoelastischen Kenngrößen waren mit beiden Anregungsmethoden vergleichbar. Elastische Eigenschaften von spezifischen anatomischen Gehirnregionen wie des Pons $\left(\left|G^{*}\right|=0,97 \pm 0,08 \mathrm{kPa}\right)$, der Capsula Interna $\left(\left|G^{*}\right|=1,29 \pm 0,14 \mathrm{kPa}\right)$ und des Crus Cerebri $\left(\left|G^{*}\right|=1,64 \pm 0,26 \mathrm{kPa}\right)$ wurden im Rahmen dieser Studie erstmals gemessen. 


\section{Diskussion}

Bisher ist die neuronale mMRE trotz vielversprechender Pilotstudien an Patienten mit Multipler Sklerose, Alzheimer, Parkinson und Gehirntumoren noch nicht in der Klinik etabliert, da unter anderem der mechanische Stimulus durch die direkte Wellenanregung am Kopf von Patienten oft als unangenehm empfunden wird. Die neue Methode erlaubt hingegen erstmalig Scherwellenanregungen ohne direkte Kopfvibration, was wesentlich zu einer Steigerung des Patientenkomforts beiträgt und längere Wiederholungsmessungen mit unterschiedlichen Anregungsfrequenzen bei reproduzierbaren Anregungsamplituden ermöglicht. Durch die beschriebenen Verbesserungen ergibt sich in künftigen Studien für die mMRE das Potential, lokale viskoelastische Veränderungen mit höherer Sensitivität zu detektieren. Die indirekte Anregungstechnik vereinfacht und verbessert die klinische Anwendbarkeit der mMRE bei neuroradiologischen Untersuchungen. 


\section{Abstract}

\section{Introduction}

Palpation is an important diagnostic method to assess elasticity changes which indicate the presence of disease processes. Using modern medical imaging methods, elastography allows quantitative evaluation of mechanical changes in living tissue. This technique enables the investigation of organs like the human brain, which cannot be palpated. Mechanical waves are generated in the brain, and the acquired wave images are used to produce maps of viscoelasticity parameters. The major goal of this study was to develop an effective and gentle indirect wave excitation setup for high-resolution cerebral multifrequency magnetic resonance elastography (mMRE) and validate it as a clinically applicable method.

\section{Methods}

The new remote wave excitation based on a thorax mat was compared to the established head rocker stimulation in 12 volunteers. A driving frequency range of 25-45 $\mathrm{Hz}$ was used for the excitation. Images were acquired in a coronal slice orientation in order to analyze specific anatomical regions along the spinothalamic pathway. A multifrequency inversion provided high-resolution maps of the viscoelastic parameters $\left|G^{*}\right|$ and $\varphi$. The amplitudes of the waves were quantified. After the measurements, the volunteers and the three operators completed a questionnaire rating the comfort and convenience of mMRE using the two methods of mechanical excitation.

\section{Results}

The remote wave excitation setup was rated more comfortable $(p=0.02)$ and easier to set up than the head rocker. Additionally, the variability of the shear wave amplitudes between the subjects, which occur with the conventional setup, was reduced. The viscoelastic parameters obtained with both setups were comparable. Specific elasticity values in the brain regions of pons $\left(\left|G^{*}\right|=0.97 \pm 0.08 \mathrm{kPa}\right)$, capsula interna $\left(\left|G^{*}\right|=1.29 \pm 0.14 \mathrm{kPa}\right)$ and crus cerebri $\left(\left|G^{*}\right|=1.64 \pm 0.26 \mathrm{kPa}\right)$ were measured in this study for the first time.

\section{Discussion}

Although pilot studies showed encouraging results in patients with multiple sclerosis, Alzheimer's disease, Parkinson's disease and brain tumors, cerebral mMRE has not been established in the clinic, due in part to discomfort from onerous mechanical stimulation applied directly 
to the head. The new method allows remote excitation, which improves patient comfort, and allows repeatable measurements with comparable wave amplitudes. As a result of these improvements, future mMRE studies have the potential to detect local viscoelasticity changes with higher sensitivity and in a wider range of patients. The indirect wave excitation setup simplifies and enhances the clinical applicability of mMRE for neuroradiological examinations. 


\section{Eidesstattliche Versicherung}

„Ich, Andreas Fehlner, versichere an Eides statt durch meine eigenhändige Unterschrift, dass ich die vorgelegte Dissertation mit dem Thema: Cerebral multifrequency MR elastography by remote excitation of intracranial shear waves selbstständig und ohne nicht offengelegte Hilfe Dritter verfasst und keine anderen als die angegebenen Quellen und Hilfsmittel genutzt habe. Alle Stellen, die wörtlich oder dem Sinne nach auf Publikationen oder Vorträgen anderer Autoren beruhen, sind als solche in korrekter Zitierung (siehe „Uniform Requirements for Manuscripts (URM)" des ICMJE - www.icmje.org) kenntlich gemacht. Die Abschnitte zu Methodik (insbesondere praktische Arbeiten, Laborbestimmungen, statistische Aufarbeitung) und Resultaten (insbesondere Abbildungen, Graphiken und Tabellen) entsprechen den URM (s. o.) und werden von mir verantwortet.

Mein Anteil an der ausgewählten Publikation entspricht dem, der in der untenstehenden gemeinsamen Erklärung mit dem Betreuer angegeben ist.

Die Bedeutung dieser eidesstattlichen Versicherung und die strafrechtlichen Folgen einer unwahren eidesstattlichen Versicherung (§156, 161 des Strafgesetzbuches) sind mir bekannt und bewusst."

Ort, Datum

Unterschrift 


\section{Ausführliche Anteilseignung an der erfolgten Publikation}

Andreas Fehlner hatte folgenden Anteil an der vorgelegten Publikation:

Andreas Fehlner, Sebastian Papazoglou, Matthew D. McGarry, Keith D. Paulsen, Jing Guo, Kaspar-Josche Streitberger, Sebastian Hirsch, Jürgen Braun, Ingolf Sack. Cerebral multifrequency MR elastography by remote excitation of intracranial shear waves. NMR in Biomedicine (Impact-Faktor 2014: 3,04). 11.2015; 28(11):1426-1432. DOI: 10.1002/nbm.3388

Beitrag im Einzelnen:

Idee und Gesamtkonzept dieser Arbeit, Literaturrecherche, Entwicklung und Optimierung der indirekten Anregungseinheit, Phantomexperimente, Anpassung der Auswertealgorithmen, Optimierung des Postprocessings, Probandenrekrutierung, Durchführung aller Versuche, vollständige Auswertung und statistische Analyse der Daten, Diskussion und Interpretation der Daten, Erstellen der ersten vollständigen Fassung des Manuskripts inklusive aller Tabellen und Abbildungen (Ausnahme: 3D-Modell des Versuchsaufbaus), eigenständige Einreichung und Korrespondenz mit dem Journal-Editor, Erstellen einer Erstfassung zur Überarbeitung des Manuskripts nach dem Review, eigenständiges Erstellen des detaillierten Response-Letters mit Darstellung der Umsetzung der Reviews, Hauptanteil der Korrektur des Manuskripts und Erstellen der Endfassung nach dem Review.

Unterschrift, Datum und Stempel des betreuenden Hochschullehrers

Unterschrift des Doktoranden 


\section{Auszug aus der Journal Summary List}

ISI Web of Knowledge ${ }^{\text {sM }}$

Journal Citation Reports ${ }^{\oplus}$

\begin{tabular}{l|lr}
\hline WELCOME & ? HELP & 2014 JCR Science Edition \\
Journal Summary List & Journal Title Changes \\
Journals from: subject categories RADIOLOGY, NUCLEAR MEDICINE \& MEDICAL IMAGING & VIEW CATEGORY SUMMARY LLST \\
Sorted by: & Impact Factor & SORT AGAIN
\end{tabular}

Journals 1 - 20 (of 125)

$\mid\langle\langle\langle\langle[|\underline{2}| \underline{3}|\underline{4}| \underline{5}|\underline{6}| \underline{7}]\rangle\rangle\rangle|$

Page 1 of 7

MARK ALL UPDATE MARKED LIST Ranking is based on your journal and sort selections.

\begin{tabular}{|c|c|c|c|c|c|c|c|c|c|c|c|}
\hline \multirow[b]{2}{*}{ Mark } & \multirow[b]{2}{*}{ Rank } & \multirow{2}{*}{$\begin{array}{c}\text { Abbreviated Journal } \\
\text { Title } \\
\text { (linked to journal } \\
\text { information) }\end{array}$} & \multirow[b]{2}{*}{ ISSN } & \multicolumn{6}{|c|}{ JCR Data i } & \multicolumn{2}{|c|}{ Eigenfactor ${ }^{\circledR}$ Metrics i } \\
\hline & & & & $\begin{array}{l}\text { Total } \\
\text { Cites }\end{array}$ & $\begin{array}{c}\text { Impact } \\
\text { Factor }\end{array}$ & \begin{tabular}{|l|} 
5-Year \\
Impact \\
Factor
\end{tabular} & $\underset{\text { Index }}{\text { Immediacy }}$ & Articles & $\begin{array}{c}\text { Cited } \\
\text { Half-life }\end{array}$ & $\begin{array}{c}\text { Eigenfactor }^{\circledR} \\
\text { Score }\end{array}$ & \begin{tabular}{|c|} 
Article \\
Influence \\
${ }^{\circledR}$ \\
Score
\end{tabular} \\
\hline$\square$ & 1 & JACC-CARDIOVASC IMAG & $1936-878 X$ & 4390 & 7.188 & 6.754 & 2.022 & 89 & 3.6 & 0.02461 & 2.777 \\
\hline$\square$ & 2 & RADIOLOGY & 0033-8419 & 48908 & 6.867 & 7.259 & 0.935 & 369 & $>10.0$ & 0.07686 & 2.548 \\
\hline$\square$ & 3 & NEUROIMAGE & $1053-8119$ & 78028 & 6.357 & 7.289 & 1.500 & 1033 & 6.5 & 0.17239 & 2.257 \\
\hline$\square$ & 4 & J NUCL MED & $0161-5505$ & 22620 & 6.160 & 6.280 & 1.208 & 308 & 7.5 & 0.04246 & 1.968 \\
\hline$\square$ & 5 & HUM BRAIN MAPP & $1065-9471$ & 16505 & 5.969 & 6.687 & 1.176 & 454 & 6.0 & 0.04232 & 2.288 \\
\hline$\square$ & 6 & CIRC-CARDIOVASC IMAG & $1941-9651$ & 2786 & 5.555 & 6.131 & 1.367 & 98 & 3.3 & 0.01759 & 2.688 \\
\hline 回 & 7 & EUR J NUCL MED MOL I & $1619-7070$ & 11724 & 5.383 & 5.090 & 1.427 & 232 & 6.1 & 0.02590 & 1.537 \\
\hline 口 & 8 & ULTRASCHALL MED & $0172-4614$ & 1476 & 4.924 & 3.468 & 0.621 & 58 & 3.5 & 0.00380 & 0.805 \\
\hline$\square$ & 9 & J CARDIOVASC MAGN R & $1097-6647$ & 2741 & 4.719 & 4.428 & 0.667 & 96 & 4.1 & 0.01145 & 1.785 \\
\hline$\square$ & 10 & INVEST RADIOL & 0020-9996 & 5463 & 4.437 & 4.418 & 0.745 & 102 & 6.8 & 0.01291 & 1.526 \\
\hline 四 & 11 & RADIOTHER ONCOL & $0167-8140$ & 12939 & 4.363 & 4.502 & 0.963 & 300 & 5.8 & 0.03164 & 1.382 \\
\hline 回 & 12 & INT J RADIAT ONCOL & $0360-3016$ & 39876 & 4.258 & 4.359 & 1.148 & 427 & 7.6 & 0.07684 & 1.389 \\
\hline$\square$ & 13 & SEMIN RADIAT ONCOL & $1053-4296$ & 1924 & 4.029 & 4.124 & 0.351 & 37 & 7.9 & 0.00411 & 1.530 \\
\hline$\square$ & 14 & EUR RADIOL & $0938-7994$ & 13516 & 4.014 & 3.735 & 0.560 & 377 & 6.1 & 0.03391 & 1.240 \\
\hline 回 & 15 & CLIN NUCL MED & $0363-9762$ & 3237 & 3.931 & 3.504 & 0.827 & 104 & 5.2 & 0.00617 & 0.800 \\
\hline$\square$ & 16 & ULTRASOUND OBST GYN & 0960-7692 & 9248 & 3.853 & 3.584 & 0.887 & 186 & 7.1 & 0.01836 & 1.127 \\
\hline$\square$ & 17 & MED IMAGE ANAL & $1361-8415$ & 4058 & 3.654 & 4.454 & 0.647 & 102 & 6.5 & 0.00847 & 1.316 \\
\hline$\square$ & 18 & BIOMED OPT EXPRESS & $2156-7085$ & 3804 & 3.648 & 3.752 & 0.656 & 346 & 2.8 & 0.01633 & 1.083 \\
\hline$\square$ & 19 & AM J NEURORADIOL & 0195-6108 & 19657 & 3.589 & 3.870 & 0.577 & 378 & 8.1 & 0.03604 & 1.269 \\
\hline$\square$ & 20 & MAGN RESON MED & 0740-3194 & 26697 & 3.571 & 3.751 & 0.763 & 426 & 9.8 & 0.03836 & 1.214 \\
\hline
\end{tabular}

MARK ALL UPDATE MARKED LIST

Journals 1 - 20 (of 125)

$\mid\langle\langle\langle\langle[1|\underline{2}| \underline{3}|\underline{4}| \underline{5}|\underline{6}| \underline{z}]\rangle \mid\rangle|$

Page 1 of 7

Acceptable Use Policy

Acceptable Use Policy
Copyright (c) 2015 Thomson Reuters.

THOMSON REUTERS 


\section{ISI Web of Knowledge ${ }^{\text {sM }}$}

\section{Journal Citation Reports ${ }^{\circledR}$}

10 WeLCOME ? HeLP

2014 JCR Science Edition

$\checkmark$ Journal Summary List

Journal Title Changes

Journals from: subject categories RADIOLOGY, NUCLEAR MEDICINE \& MEDICAL IMAGING GIEW CATEGORY SUMMARY LIST

Sorted by: Impact Factor SORT AGAIN

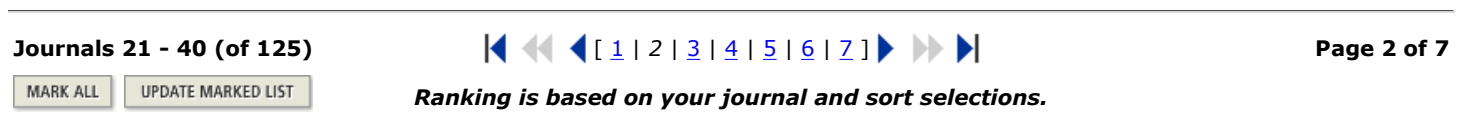

\begin{tabular}{|c|c|c|c|c|c|c|c|c|c|c|c|}
\hline \multirow[b]{2}{*}{ Mark } & \multirow[b]{2}{*}{ Rank } & \multirow{2}{*}{$\begin{array}{c}\text { Abbreviated Journal } \\
\text { Title } \\
\text { (linked to journal } \\
\text { information) }\end{array}$} & \multirow[b]{2}{*}{ ISSN } & \multicolumn{6}{|c|}{ JCR Data i } & \multicolumn{2}{|c|}{ Eigenfactor $^{\circledR}$ Metrics i } \\
\hline & & & & $\begin{array}{l}\text { Total } \\
\text { Cites }\end{array}$ & $\begin{array}{l}\text { Impact } \\
\text { Factor }\end{array}$ & $\begin{array}{c}\text { 5-Year } \\
\text { Impact } \\
\text { Factor }\end{array}$ & $\begin{array}{c}\text { Immediacy } \\
\text { Index }\end{array}$ & Articles & $\begin{array}{c}\text { Cited } \\
\text { Half-life }\end{array}$ & $\begin{array}{c}\text { Eigenfactor }^{\circledR} \\
\text { Score }\end{array}$ & $\begin{array}{c}\text { Article } \\
\text { Influence }{ }^{\circledR} \\
\text { Score }\end{array}$ \\
\hline$\square$ & 21 & IEEE T MED IMAGING & $0278-0062$ & 12720 & 3.390 & 4.288 & 0.435 & 184 & 9.8 & 0.02070 & 1.437 \\
\hline$\square$ & 22 & SEMIN NUCL MED & 0001-2998 & 1853 & 3.342 & 3.751 & 1.175 & 40 & 7.3 & 0.00348 & 1.159 \\
\hline$\square$ & 23 & J MAGN RESON IMAGING & $1053-1807$ & 13539 & 3.210 & 3.250 & 0.523 & 363 & 6.6 & 0.03312 & 1.163 \\
\hline$\square$ & 24 & NMR BIOMED & $0952-3480$ & 5642 & 3.044 & 3.529 & 0.372 & 164 & 6.6 & 0.01527 & 1.325 \\
\hline$\square$ & 25 & $\underline{Z}$ MED PHYS & 0939-3889 & 362 & 2.963 & 1.896 & 0.733 & 30 & 3.0 & 0.00123 & 0.578 \\
\hline$\square$ & 26 & J NUCL CARDIOL & $1071-3581$ & 2574 & 2.942 & 2.664 & 0.755 & 94 & 5.6 & 0.00625 & 0.802 \\
\hline$\square$ & 27 & CONTRAST MEDIA MOL I & $1555-4309$ & 944 & 2.923 & 2.945 & 0.886 & 44 & 3.5 & 0.00348 & 0.920 \\
\hline$\square$ & 28 & STRAHLENTHER ONKOL & 0179-7158 & 2522 & 2.914 & 2.402 & 0.728 & 147 & 4.9 & 0.00439 & 0.505 \\
\hline$\square$ & 29 & $\underline{\text { RADIAT RES }}$ & 0033-7587 & 8207 & 2.911 & 2.880 & 0.565 & 131 & $>10.0$ & 0.01080 & 0.851 \\
\hline$\square$ & 30 & MAGN RESON MATER PHY & $0968-5243$ & 1189 & 2.869 & 2.594 & 0.255 & 55 & 6.2 & 0.00360 & 1.069 \\
\hline$\square$ & 31 & J BIOMED OPT & $1083-3668$ & 11293 & 2.859 & 2.926 & 0.519 & 499 & 4.8 & 0.02738 & 0.762 \\
\hline$\square$ & 32 & J AM COLL RADIOL & $1546-1440$ & 1863 & 2.836 & & 0.769 & 147 & 3.9 & 0.00644 & \\
\hline$\square$ & 33 & MOL IMAGING BIOL & $1536-1632$ & 1814 & 2.774 & 2.764 & 0.604 & 96 & 3.9 & 0.00635 & 0.890 \\
\hline$\square$ & 34 & PHYS MED BIOL & 0031-9155 & 20131 & 2.761 & 2.973 & 0.450 & 460 & 7.4 & 0.04082 & 0.971 \\
\hline$\square$ & 35 & BRACHYTHERAPY & $1538-4721$ & 1115 & 2.758 & 2.169 & 0.696 & 92 & 3.3 & 0.00324 & 0.596 \\
\hline$\square$ & 36 & AM J ROENTGENOL & 0361-803x & 29458 & 2.731 & 3.302 & 0.447 & 494 & 9.2 & 0.04876 & 1.086 \\
\hline$\square$ & 37 & INT J HYPERTHER & $0265-6736$ & 2247 & 2.645 & 2.469 & 0.357 & 70 & 6.8 & 0.00408 & 0.658 \\
\hline$\square$ & 38 & MED PHYS & 0094-2405 & 20845 & 2.635 & 2.954 & 0.363 & 593 & 6.6 & 0.04256 & 0.859 \\
\hline$\square$ & 39 & RADIOGRAPHICS & 0271-5333 & 8478 & 2.602 & 3.807 & 0.262 & 122 & 8.9 & 0.01271 & 1.233 \\
\hline 四 & 40 & RADIAT ONCOL & $1748-717 X$ & 2889 & 2.546 & 2.749 & 0.416 & 269 & 3.0 & 0.01196 & 0.844 \\
\hline
\end{tabular}

MARK ALL UPDATE MARKED LIST

Journals 21 - 40 (of 125)

$\mid\langle\langle[\underline{1}|2| \underline{3}|\underline{4}| \underline{5}|\underline{6}| \underline{7}]\rangle \Rightarrow\rangle$

Page 2 of 7

Acceptable Use Policy

Copyright (c) 2015 Thomson Reuters.

THOMSON REUTERS

Published by Thomson Reuters

Das Journal NMR in Biomedicine ist nach dem ISI Journal Citation Reports Ranking 2014 im Bereich Radiology Nuclear Medicine $\&$ Medical Imaging auf Position 24/125, Top $20 \%$ (>30\%) und hat einen Eigenfaktor von 0,01527 (>0,01). 


\section{Druckexemplar der ausgewählten Publikation}

Die Seiten 11-17 umfassen den folgenden Originalartikel:

Andreas Fehlner, Sebastian Papazoglou, Matthew D. McGarry, Keith D. Paulsen, Jing Guo, Kaspar-Josche Streitberger, Sebastian Hirsch, Jürgen Braun, Ingolf Sack. Cerebral multifrequency MR elastography by remote excitation of intracranial shear waves. NMR in Biomedicine (Impact-Faktor 2014: 3,04). 11.2015; 28(11):1426-1432. http://dx.doi.org/10.1002/nbm.3388 


\section{Lebenslauf}

Der Lebenslauf ist in der Online-Version aus Gründen des Datenschutzes nicht enthalten. 


\section{Publikationsliste}

Seit Beginn meiner Tätigkeit als wissenschaftlicher Mitarbeiter in der AG Elastographie (Charité - Universitätsmedizin Berlin) entstanden die folgenden Originalarbeiten und Konferenzbeiträge unter meiner Erst- oder Mitautorenschaft:

\section{Originalarbeiten}

1. Kaspar-Josche Streitberger, Andreas Fehlner, Florence Pache, Anna Lacheta, Sebastian Papazoglou, Judith Bellmann-Strobl, Klemens Ruprecht, Alexander Brandt, Jürgen Braun, Ingolf Sack, Friedemann Paul, Jens Wuerfel. Multifrequency magnetic resonance elastography of the brain reveals tissue degeneration in neuromyelitis optica spectrum disorder. European Radiology. 08.2016; DOI: 10.1007/s00330-016-4561-6

2. Andreas Fehlner, Janina R. Behrens, Kaspar-Josche Streitberger, Sebastian Papazoglou, Jürgen Braun, Judith Bellmann-Strobl, Klemens Ruprecht, Friedemann Paul, Jens Würfel, Ingolf Sack. Higher resolution MR elastography reveals early mechanical signatures of neuroinflammation in patients with clinically isolated syndrome. Journal of Magnetic Resonance Imaging. 07.2016; 44(1):51-58. DOI: 10.1002/jmri.25342

3. Andreas Fehlner, Sebastian Papazoglou, Matthew D. McGarry, Keith D. Paulsen, Jing Guo, Kaspar-Josche Streitberger, Sebastian Hirsch, Jürgen Braun, Ingolf Sack. Cerebral multifrequency MR elastography by remote excitation of intracranial shear waves. NMR in Biomedicine. 11.2015; 28(11):1426-1432. DOI: 10.1002/nbm.3388

4. Kaspar-Josche Streitberger, Gerd Diederichs, Jing Guo, Andreas Fehlner, Bernd Hamm, Jürgen Braun, Ingolf Sack. In vivo multifrequency magnetic resonance elastography of the human intervertebral disk. Magnetic Resonance in Medicine. 11.2015; 74(5):1380-1387. DOI: 10.1002/mrm.25505

5. Seyed R. Mousavi, Andreas Fehlner, Kaspar-Josche Streitberger, Jürgen Braun, Abbas Samani, Ingolf Sack. Measurement of in vivo cerebral volumetric strain induced by the Valsalva maneuver. Journal of Biomechanics. 05.2014; 47(7):1652-1657. DOI: 10.1016/j.jbiomech.2014.02.038

6. Jing Guo, Sebastian Hirsch, Andreas Fehlner, Sebastian Papazoglou, Michael Scheel, Jürgen Braun, Ingolf Sack. Towards an elastographic atlas of brain anatomy. PLoS ONE. 01.2013; 8(8):e71807. DOI: 10.1371/journal.pone.0071807

7. Axel Lipp, Radmila Trbojevic, Friedemann Paul, Andreas Fehlner, Sebastian Hirsch, Michael Scheel, Cornelia Noack, Jürgen Braun, Ingolf Sack. Cerebral magnetic resonance elastography in supranuclear palsy and idiopathic Parkinson's disease. NeuroImage: Clinical. 01.2013; 3:381-387. DOI: 10.1016/j.nicl.2013.09.006 


\section{Konferenzbeiträge}

1. Patric Birr, Andreas Fehlner, Sebastian Hirsch, Florian Dittmann, Jing Guo, Jürgen Braun, Ingolf Sack, Stefan Hetzer. Cerebral tissue characterization by magnetic resonance elastography and arterial spin labeling. Proceedings of the 24th Annual Meeting ISMRM. Singapore; 2016: 3320

2. Andreas Fehlner, Sebastian Hirsch, Mykola Kadobianskyi, Patric Birr, Eric Barnhill, Martin Weygandt, Johannes Bernarding, Jürgen Braun, Ingolf Sack, Stefan Hetzer. Increasing the spatial resolution and sensitivity of high-resolution magnetic resonance elastography by correcting for subject motion and susceptibility-induced image distortions. Proceedings of the 24th Annual Meeting ISMRM. Singapore; 2016: 1945

3. Jing Guo, Stephan Marticorena, Florian Dittmann, Andreas Fehlner, Sebastian Hirsch, Thomas Fischer, Jürgen Braun, Ingolf Sack. Magnetic resonance elastography (MRE) for the assessment of renal allograft function. Proceedings of the 24th Annual Meeting ISMRM. Singapore; 2016: 0707

4. Jing Guo, Christian Hudert, Heiko Tzschätzsch, Andreas Fehlner, Florian Dittmann, Jürgen Braun, Ingolf Sack. Multifrequency MR elastography for assessing hepatic fibrosis in pediatric non-alcoholic fatty liver disease. Proceedings of the 24th Annual Meeting ISMRM. Singapore; 2016: 0362

5. Andreas Fehlner, Lea Gerischer, Agnes Flöel, Jürgen Braun, Ingolf Sack. High resolution MR elastography of the hippocampus reveals differential tissue elasticity in Alzheimer's disease - a pilot study. Proceedings of the 24th Annual Meeting ISMRM. Singapore; 2016: 0148

6. Lea M. Gerischer, Andreas Fehlner, Theresa Köbe, Jürgen Braun, Ingolf Sack, Agnes Flöel. High resolution MR elastography of the hippocampus as a novel biomarker for Alzheimer's disease? Clinical Neurophysiology. 09.2016; 127(9):e216-e217. DOI: 10.1016/j.clinph.2016.05.029

7. Kaspar-Josche Streitberger, Andreas Fehlner, Friedemann Paul, Jens Würfel, Jing Guo, Jürgen Braun, Ingolf Sack. High resolution MR elastography reveals retrograde thalamic tissue degradation in neuromyelitis optica. Proceedings of the 23rd Annual Meeting ISMRM. Toronto, ON, Canada; 2015: 2211

8. Andreas Fehlner, Kaspar-Josche Streitberger, Friedemann Paul, Jens Würfel, Jürgen Braun, Ingolf Sack. High resolution MR elastography reveals disseminated white matter degradation of brain tissue integrity in clinically isolated syndrome. Proceedings of the 23rd Annual Meeting ISMRM. Toronto, ON, Canada; 2015: 0820 
9. Jing Guo, Sebastian Hirsch, Sebastian Papazoglou, Kaspar-Josche Streitberger, Andreas Fehlner, Jürgen Braun, Ingolf Sack. Magnetic resonance elastography of cysts and fluid filled cavities. Proceedings of the 22nd Annual Meeting ISMRM. Milan, Italy; 2014: 1696

10. Andreas Fehlner, Sebastian Papazoglou, Jing Guo, Kaspar-Josche Streitberger, Sebastian Hirsch, Jürgen Braun, Ingolf Sack. Remotely induced cerebral strain for enhanced safety and acceptance of MR elastography of the brain. Proceedings of the 22nd Annual Meeting ISMRM. Milan, Italy; 2014: 1693

11. Kaspar-Josche Streitberger, Jing Guo, Gerd Diederichs, Sebastian Hirsch, Andreas Fehlner, Jürgen Braun, Ingolf Sack. In vivo magnetic resonance elastography of the human intervertebral disk: preliminary results. Proceedings of the 22nd Annual Meeting ISMRM. Milan, Italy; 2014: 1244

12. Andreas Fehlner, Sebastian Hirsch, Jing Guo, Jürgen Braun, Ingolf Sack. The viscoelastic response of the human brain to functional activation detected by magnetic resonance elastography. Proceedings of the 22nd Annual Meeting ISMRM. Milan, Italy; 2014: 0871

13. Jürgen Braun, Ralf Lützkendorf, Jing Guo, Sebastian Hirsch, Andreas Fehlner, Ingolf Sack, Johannes Bernarding. High resolution 3D multifrequency MR elastography at 7T. Proceedings of the 21st Annual Meeting ISMRM. Salt Lake City, USA; 2013: 2439

14. Ingolf Sack, Rolf Reiter, Korinna Joehrens, Andreas Fehlner, Sebastian Hirsch, Jing Guo, Rajan Somasundaram, Daniel Seehofer, Carsten Kamphues, Abbas Samani, Jürgen Braun. Multifrequency MRE of human liver specimen: sensitivity of viscoelastic powerlaw constants to the collagen matrix in hepatic fibrosis. Proceedings of the 21st Annual Meeting ISMRM. Salt Lake City, USA; 2013: 2428

15. Jing Guo, Sebastian Hirsch, Sebastian Papazoglou, Andreas Fehlner, Michael Scheel, Jens Würfel, Jürgen Braun, Ingolf Sack. An atlas of the anatomy of human brain viscoelasticity. Proceedings of the 21st Annual Meeting ISMRM. Salt Lake City, USA; 2013: 0873

16. Ingolf Sack, Axel Lipp, Radmila Trbojevic, Friedemann Paul, Andreas Fehlner, Sebastian Hirsch, Michael Scheel, Cornelia Noack, Jing Guo, Dieter Klatt, Jens Würfel, Jürgen Braun. Brain softening - a hallmark of neurodegeneration: cerebral MR elastography in patients with supranuclear palsy and idiopathic Parkinson's disease. Proceedings of the 21st Annual Meeting ISMRM. Salt Lake City, USA; 2013: 0380

17. Sebastian Hirsch, Frauke Beyer, Jing Guo, Sebastian Papazoglou, Heiko Tzschätzsch, Andreas Fehlner, Ingolf Sack, Jürgen Braun. Compression sensitive magnetic reso- 
nance elastography. Proceedings of the 11th International Tissue Elasticity Conference. Deauville, France; 2012: 48

18. Andreas Fehlner, Sebastian Hirsch, Jürgen Braun, Ingolf Sack. Fast 3D vector field multi-frequency magnetic resonance elastography of the human brain - preliminary results. Bildverarbeitung für die Medizin. Berlin, Germany; 2012. DOI: 10.1007/978-3642-28502-8_63

\section{Auszeichnungen}

ISMRM Merit Award Summa Cum Laude von der International Society for Magnetic Resonance in Medicine für den Konferenzbeitrag

Andreas Fehlner, Kaspar-Josche Streitberger, Friedemann Paul, Jens Würfel, Jürgen Braun, Ingolf Sack. High resolution MR elastography reveals disseminated white matter degradation of brain tissue integrity in clinically isolated syndrome. Proceedings of the 23rd Annual Meeting ISMRM. Toronto, ON, Canada; 2015: 0820

ISMRM Merit Award Magna Cum Laude von der International Society for Magnetic Resonance in Medicine für die Konferenzbeiträge

Andreas Fehlner, Sebastian Hirsch, Mykola Kadobianskyi, Patric Birr, Eric Barnhill, Martin Weygandt, Johannes Bernarding, Jürgen Braun, Ingolf Sack, Stefan Hetzer. Increasing the spatial resolution and sensitivity of high-resolution magnetic resonance elastography by correcting for subject motion and susceptibility-induced image distortions. Proceedings of the 24th Annual Meeting ISMRM. Singapore; 2016: 1945

Andreas Fehlner, Lea Gerischer, Agnes Flöel, Jürgen Braun, Ingolf Sack. High resolution MR elastography of the hippocampus reveals differential tissue elasticity in Alzheimer's disease - a pilot study. Proceedings of the 24th Annual Meeting ISMRM. Singapore; 2016: 0148

2. Platz in der Kategorie Best Oral Presentation der White Matter Study Group auf dem Annual Meeting der International Society for Magnetic Resonance in Medicine 2016 in Singapur.

Andreas Fehlner, Lea Gerischer, Agnes Flöel, Jürgen Braun, Ingolf Sack. High resolution MR elastography of the hippocampus reveals differential tissue elasticity in Alzheimer's disease - a pilot study. Proceedings of the 24th Annual Meeting ISMRM. Singapore; 2016: 0148 


\section{Danksagung}

Bei allen, welche mich bei der Umsetzung der verschiedenen Projekte unterstützt haben, möchte ich mich herzlich bedanken.

Mein besonderer Dank gilt Herrn Prof. Dr. Ingolf Sack und Herrn PD Dr. Jürgen Braun, die es mir ermöglicht haben, in einem sehr innovativen medizinphysikalischen Forschungsbereich an verschiedenen interessanten wissenschaftlichen Kooperationsprojekten teilzunehmen und eigene Projekte zu entwickeln. Ihre wertvollen Ratschläge und ihre ständige Ansprechbarkeit trugen entscheidend zum Gelingen der Arbeit bei.

Ich möchte mich weiterhin bei den Mitgliedern der AG Elastographie (Charité - Universitätsmedizin Berlin), insbesondere bei B.Sc. Patric Birr, M.Sc. Florian Dittmann, Dr. Jing Guo, Dr. Sebastian Hirsch, M.Sc. Selcan Ipek-Ugay, Dipl.-Ing. M.Sc. Dirk Lohse, Dr. Sebastian Papazoglou und Dipl.-Phys. Heiko Tzschätzsch sehr für die herzliche Atmosphäre bedanken. Zudem möchte ich ihnen und allen weiteren Freiwilligen, die in den verschiedenen Projekten als Probanden meine Studien unterstützt haben, danken.

Bedanken möchte ich mich außerdem bei der Hanns-Seidel-Stiftung, die meine Arbeit durch ein Promotionsstipendium sowohl ideell als auch finanziell unterstützt hat. Durch diese Unterstützung war es mir auch möglich, an sehr spannenden und informativen Seminaren und anderen Veranstaltungen teilzunehmen. 